\title{
COLLIDING PLANE WAVES IN EINSTEIN-MAXWELL-DILATON FIELDS
}

\author{
Nora Bretón, Tonatiuh Matos and Alberto García \\ Department of Physics \\ CINVESTAV, A.P. 14-740, 07000,MEXICO,D.F.
}

\begin{abstract}
Within the metric structure endowed with two orthogonal space-like Killing vectors a class of solutions of the Einstein-Maxwell-Dilaton field equations is presented. Two explicitly given sub-classes of solutions bear an interpretation as colliding plane waves in the low-energy limit of the heterotic string theory.
\end{abstract}

PACS number(s) : 04.50.th, 04.40.Nr, 11.25.Mj 


\section{Introduction}

The study of the gravitational interaction coupled to the Maxwell and dilaton fields has been the subject of recent investigations related to the heterotic string theory. Dilaton fields coupled to Einstein-Maxwell fields appear in a natural manner in the low-energy effective action in string theory and as a result of a dimensional reduction of the KaluzaKlein Lagrangian. It has been realized that the low-energy effective field, which describes string theory, contains solutions endowed with qualitatively different features from those ones that appear in ordinary Einstein gravity [1].

Lately it has been found that plane wave geometries are exact solutions for the string theory to all orders of string tension parameter [2]. It is therefore of interest to consider the collision of plane gravitational waves with electromagnetic and dilaton fields. In fact, some solutions of this kind have been already presented by Gürses [3].

In the context of General Relativity the topic of colliding plane gravitational waves has been widely explored and colliding wave solutions with scalar fields have been found too. However, those scalar fields were weakly coupled to the electromagnetic field [4], while the most intriguing features of string gravity are due to the peculiar nature of the dilaton heterotic coupling to vector fields. Here we consider the stringy gravity model including vector fields for colliding plane gravitational waves, i. e., the Einstein-Maxwell-Dilaton (EMD) system with an arbitrary dilaton coupling constant in the framework of interacting plane waves.

We consider the action [5]

$$
S=\int d^{4} x \sqrt{-g}\left\{-R+2(\nabla \Phi)^{2}+e^{-2 \alpha \Phi} F^{2}\right\},
$$

where $g=\operatorname{det}\left(g_{\mu \nu}\right), \mu, \nu=0,1,2,3 . R$ is the scalar curvature, $F_{\mu \nu}$ is the Maxwell field, and $\Phi$ is the dilaton field. The constant $\alpha$ is a free parameter which governs the strength of the coupling of the dilaton to the Maxwell field. Special theories are contained in (1): For $\alpha=\sqrt{3}$, the action (1) leads to the Kaluza- Klein field equations obtained from the dimensional reduction of the five- dimensional Einstein vacuum equations. For $\alpha=1$, 
the action (1) coincides with the low energy limit of string theory with vanishing dilaton potential [6]. Finally, in the extreme limit $\alpha=0$, (1) yields the Einstein-Maxwell theory minimally coupled to the scalar field.

The field equations obtained from (1) are

$$
\begin{gathered}
\left(e^{-2 \alpha \Phi} F^{\mu \nu}\right)_{; \mu}=0, \\
\Phi_{; \mu}^{; \mu}+\frac{\alpha}{2} e^{-2 \alpha \Phi} F_{\mu \nu} F^{\mu \nu}=0 \\
R_{\mu \nu}=2 \Phi_{, \mu} \Phi_{, \nu}+2 e^{-2 \alpha \Phi}\left(F_{\mu \lambda} F_{\nu}^{\lambda}-\frac{1}{4} g_{\mu \nu} F_{\alpha \beta} F^{\alpha \beta}\right),
\end{gathered}
$$

where a semicolon denotes the covariant derivative with respect to $g_{\mu \nu}$. A few exact solutions of Eqs. (2)-(4) are known; they reveal many interesting features of the dilaton field (see [1] and references therein). In this paper we present solutions to Eqs. (2)-(4) with a colliding plane wave interpretation. We first present the solutions in the interaction region and then extend them beyond the null boundaries. In the next section we outline the usual representation of the colliding plane wave spacetime in General Relativity and the corresponding field equations. In section 3 we present explicitly the solutions and check that the appropriate boundary conditions for colliding waves are satisfied. In section 4 we comment about the nature of the singularity and finaly we draw some conclusions in section 5 .

\section{The Colliding Waves Spacetime and the Field Equations}

A spacetime describing the collision of plane waves admits two spacelike Killing vector fields. In this work we take them to be orthogonal. For such a case, we consider the metric $g_{\mu \nu}$ and the $\mathrm{U}(1)$ gauge potential $A_{\mu}$ as given by

$$
\begin{gathered}
d s^{2}=2 e^{-M} d u d v+e^{-U}\left(e^{-V} d y^{2}+e^{V} d x^{2}\right), \\
A_{\mu}=(0,0, A, 0)
\end{gathered}
$$

where $M=M(u, v), U=U(u, v), V=V(u, v), A=A(u, v)$ and the electromagnetic field is $F_{\mu \nu}=A_{\nu, \mu}-A_{\mu, \nu}$ 
The spacetime for the collision of plane waves is divided into four disjoint regions: Region I (of interaction): $0 \leq u \leq 1, \quad 0 \leq v \leq 1$. Region II : $u<0, \quad 0<v<1$; and Region III : $0<u<1, \quad v<0$, where "live" the incoming waves. The boundaries between the region I and regions II and III are $u=0$ and $v=0$. Finally, it is considered the region IV $: u<0, \quad v<0$, which corresponds to the spacetime before the pass of any wave. The line element (5) applies to the entire spacetime, however the metric functions $U, V$ and $M$ must take different forms in the four regions.

The field equations (2)-(4) turn out to be

$$
\begin{gathered}
-2 A_{, u v}=\left(V_{, u}-\alpha \Phi_{, u}\right) A_{, v}+\left(V_{, v}-\alpha \Phi_{, v}\right) A_{, u} \\
U_{, u v}=U_{, u} U_{, v} \quad, \\
2 M_{, u v}=-2 U_{, u v}+U_{, u} U_{, v}+V_{, u} V_{, v}+4 \Phi_{, u} \Phi_{, v} \quad, \\
2 V_{, u v}-U_{, u} V_{, v}-U_{, v} V_{, u}-4 e^{U+V-\alpha \Phi} A_{, u} A_{, v}=0, \\
2 \Phi_{, u v}-U_{, u} \Phi_{, v}-U_{, v} \Phi_{, u}+\frac{\alpha}{2} e^{U+V-\alpha \Phi} A_{, u} A_{, v}=0, \\
-2 M_{, u} U_{, u}-2 U_{, u u}+U_{, u}^{2}+V_{, u}^{2}+4 \Phi_{, u}^{2}+4 e^{U+V-\alpha \Phi} A_{, u}^{2}=0 \\
-2 M_{, v} U_{, v}-2 U_{, v v}+U_{, v}^{2}+V_{, v}^{2}+4 \Phi_{, v}^{2}+4 e^{U+V-\alpha \Phi} A_{, v}^{2}=0,
\end{gathered}
$$

The dilaton field $\Phi=\Phi(u, v)$. Note that Eq. (9) can be derived from the other equations. Eq. (8) can be immediately integrated

$$
e^{-U}=a(u)+b(v)
$$

with $a$ and $b$ being arbitrary functions of $u$ and $v$ respectively.

The corresponding components of the Weyl tensor are computed to be 


$$
\begin{gathered}
\Psi_{o}^{o}=-\frac{1}{2}\left[V_{, v v}-V_{, v}\left(U_{, v}-M_{, v}\right)\right], \\
\Psi_{4}^{o}=-\frac{1}{2}\left[V_{, u u}-V_{, u}\left(U_{, u}-M_{, u}\right)\right], \\
\Psi_{2}^{o}=\frac{1}{2} M_{, u v}, \quad \Psi_{1}^{o}=\Psi_{3}^{o}=0,
\end{gathered}
$$

We shall give in the next section the solution for region I and then we discuss the matching to the precolliding regions.

\section{The EMD solutions}

Although one can proceed with the above $(u, v)$-dependence formulation, it occurs more effective -from the integration point of view- to use a $(\rho, z)$-dependence, i. e., to look for solutions for the EMD Eqs. (2)-(4) for a diagonal line element of the form

$$
d s^{2}=\frac{e^{2 k}}{f}\left(d \rho^{2}-d z^{2}\right)+\rho\left[\rho f^{-1} d x^{2}+\rho^{-1} f d y^{2}\right]
$$

with $\partial_{x}$ and $\partial_{y}$ being the two commuting spacelike Killing vectors, and $f, k$ being functions of $\rho$ and $z$ only. We can arrive to (18) from the metric (5) by defining

$$
\begin{aligned}
& \rho=e^{-U}=a(u)+b(v), \\
& z=a(u)-b(v),
\end{aligned}
$$

and identifying $2 k \rightarrow-(M+V+U)-\ln \left[2 a^{\prime}(u) b^{\prime}(v)\right]$ and $f \rightarrow \exp [-(V+U)]$, where $a^{\prime}(u)$ and $b^{\prime}(v)$ denote the derivatives in $u$ and $v$ respectively.

The method used to determine the sought solutions is the harmonic mapping combined with the algebra associated to the group $S L(2, \mathbf{R})$, which reduce the integration of the Einstein's equations to an algebraic problem (see [7] and references therein). Other methods to obtain solutions have been addressed like the inverse scattering method [8], however, we encounter that by means of the harmonic map one gets wider class of solutions in a more straightforward manner.

A class of solutions for the EMD Eqs. (2)-(4) is given by 


$$
\begin{gathered}
f=\frac{f_{o} e^{\lambda}}{\left(a_{1} \Sigma_{1}+a_{2} \Sigma_{2}\right)^{\gamma}}, \\
\kappa^{2}=e^{-2 \alpha \Phi}=\kappa_{o}^{2}\left(a_{1} \Sigma_{1}+a_{2} \Sigma_{2}\right)^{\beta} e^{\lambda-\tau_{o} \tau} \\
A=A_{y}=\frac{\left(a_{3} \Sigma_{1}+a_{4} \Sigma_{2}\right)}{\left(a_{1} \Sigma_{1}+a_{2} \Sigma_{2}\right)}
\end{gathered}
$$

where $\Sigma_{1}$ and $\Sigma_{2}$ denote functions on the variable $\tau(\rho, z)$ which is determined by the harmonic map (Eq.(14) in Ref. [7]); for each pair $\left(\Sigma_{1}, \Sigma_{2}\right)$ we have a different solution for the Eqs. (20)-(22) (See Eqs.(25) and (27) below); $\tau_{o}, \kappa_{o}, f_{o}, a_{1}, a_{2}, a_{3}$, and $a_{4}$ are constants, and $\gamma$ and $\beta$ are $\alpha$-dependent parameters

$$
\gamma=\frac{2}{1+\alpha^{2}}, \quad \beta=\frac{2 \alpha^{2}}{1+\alpha^{2}}
$$

The functions $\lambda(\rho, z)$ and $\tau(\rho, z)$ are each one a solution of the equation

$$
\phi_{, \rho \rho}+\frac{1}{\rho} \phi_{, \rho}-\phi_{, z z}=0
$$

Among the above solutions (20)-(22), we distinguish two cases

case $(i)$

$$
\Sigma_{1}=e^{q_{1} \tau}, \quad \Sigma_{2}=e^{q_{2} \tau}, \quad 4 a_{1} a_{2} f_{o}+\kappa_{o}^{2}\left(1+\alpha^{2}\right)\left(a_{1} a_{4}-a_{3} a_{2}\right)^{2}=0
$$

where $q_{1}$ and $q_{2}$ are constants. The corresponding equations for $k$, the transversal gravitational degree of freedom, are

$$
\begin{aligned}
& k_{, z}=\frac{\rho}{2}\left\{\left(\frac{\alpha^{2}+1}{\alpha^{2}}\right) \lambda_{, \rho} \lambda_{, z}-\left(2 \gamma q_{1} q_{2}-\frac{\tau_{o}^{2}}{\alpha^{2}}\right) \tau_{, \rho} \tau_{, z}-\frac{\tau_{o}}{\alpha^{2}}\left(\tau_{, z} \lambda_{, \rho}+\tau_{, \rho} \lambda_{, z}\right)\right\}, \\
& k_{, \rho}=\frac{\rho}{4}\left\{\left(\frac{\alpha^{2}+1}{\alpha^{2}}\right)\left(\lambda_{, \rho}^{2}+\lambda_{, z}^{2}\right)-\left(2 \gamma q_{1} q_{2}-\frac{\tau_{o}^{2}}{\alpha^{2}}\right)\left(\tau_{, \rho}^{2}+\tau_{, z}^{2}\right)-\frac{2 \tau_{o}}{\alpha^{2}}\left(\tau_{, z} \lambda_{, z}-\tau_{, \rho} \lambda_{, \rho}\right)\right\},
\end{aligned}
$$

which are integrable once one specifies $\lambda(\rho, z)$ and $\tau(\rho, z)$, solutions of Eq. (24).

case $(i i)$

$$
\Sigma_{1}=\tau, \quad \Sigma_{2}=1, \quad q_{1}=-q_{2}, \quad 4 a_{1}^{2} f_{o}-\kappa_{o}^{2}\left(1+\alpha^{2}\right)\left(a_{1} a_{4}-a_{3} a_{2}\right)^{2}=0,
$$

The corresponding equations for $k$ are 


$$
\begin{aligned}
& k_{, z}=\rho \lambda_{, \rho} \lambda_{, z}, \\
& k_{, \rho}=\frac{\rho}{4}\left(\lambda_{, \rho}^{2}+\lambda_{, z}^{2}\right) .
\end{aligned}
$$

which, again, are integrable as soon as one specifies $\lambda(\rho, z)$, solution of Eq. (24).

The solutions of Eq. (24) are of the form

$$
\begin{aligned}
\phi= & K \ln \rho+L\left\{A_{\omega} \cos \left[\omega\left(z+z_{o}\right)\right] J_{o}(\omega \rho)\right\}+ \\
& L\left\{B_{\omega} \cos \left[\omega\left(z+z_{o}\right)\right] N_{o}(\omega \rho)\right\}-\sum_{i} d_{i} \operatorname{arccosh}\left(\frac{z+z_{i}}{\rho}\right),
\end{aligned}
$$

where $K$ is a constant, $L\{\}$ stands for arbitrary linear combinations of the terms in curly brackets and $J_{o}(\omega \rho)$ and $N_{o}(\omega \rho)$ are the Bessel and Neumann functions of zero order respectively.

An explicit relationship between the coordinates $(\rho, z)$ of the metric (18) and the null coordinates $(u, v)$ of the metric (5) is given when we select $a(u)=\frac{1}{2}-u^{n}, \quad b(v)=\frac{1}{2}-v^{m}$, then we have

$$
\rho=1-u^{n}-v^{m}, \quad z=v^{m}-u^{n}
$$

with $m$ and $n$ being constants determined by boundary conditions. The null coordinates $(u, v)$ are more suitable for the analysis of the matching conditions, which we address in the next subsection.

\section{Continuity of the Metric on the Null Boundary}

The solutions for cases $(i)$ and $(i i)$ can be interpreted as the gravitational field in the interaction region arising after the collision of two gravitational plane waves only if certain boundary conditions on the null hypersurfaces $u=0$ and $v=0$ are satisfied [9]. With the chosen coordinate relation, Eq.(30), one has to verify only the continuity on $u=0$ and $v=0$ of the metric coefficient $g_{u v}=4 m n u^{n-1} v^{m-1} e^{2 k} f^{-1}$, which arises when we substitute Eq. (30) in (18), and taking the expression for $f$, Eq. (20), one arrives at

$$
g_{u v}=4 m n u^{n-1} v^{m-1} e^{2 k} f_{o}^{-1}\left\{a_{1} \Sigma_{1}+a_{2} \Sigma_{2}\right\}^{\gamma} e^{-\lambda} .
$$


We shall prove separately the continuity on $u=0$ and $v=0$ of the appearing above factors

$$
\begin{aligned}
& \left(a_{1} \Sigma_{1}+a_{2} \Sigma_{2}\right)^{\gamma} e^{-\lambda}, \\
& \text { and } u^{n-1} v^{m-1} e^{2 k} .
\end{aligned}
$$

For the case $(i)$, without loss of generality, we can take as solutions for $\tau$ and $\lambda$ the following functions [10]

$$
\begin{aligned}
& \tau=d_{1} \operatorname{arccosh}\left[\frac{z+1}{\rho}\right]=d_{1} \ln \left[\frac{z+1 \pm \sqrt{(z+1)^{2}-\rho^{2}}}{\rho}\right] \\
& \lambda=d_{2} \operatorname{arccosh}\left[\frac{1-z}{\rho}\right]=d_{2} \ln \left[\frac{1-z \pm \sqrt{(1-z)^{2}-\rho^{2}}}{\rho}\right]
\end{aligned}
$$

where $d_{1}$ and $d_{2}$ are constants. Substituting the expressions (34) into (32) and taking separately the limits $u \rightarrow 0$ and $v \rightarrow 0$ (noting that $u=0$ corresponds to $\rho=-z+1$ while $v=0$ corresponds to $\rho=z+1$ ), it is easy to see that the factor (32) does not diverge on $u=0$ neither on $v=0$. Thus we are led with the factor $(33)$, i. e., $u^{n-1} v^{m-1} e^{2 k}$. To ensure the smooth matching between the interaction and the precollision regions, the function $e^{2 k}$ must diverge as $u^{1-n}$ and $v^{1-m}$ on $u=0$ and $v=0$ respectively. This divergence in $e^{2 k}$ comes from the terms of Eqs. (34); to show that, we note from Eqs. (26) that one can split the function $k$ as

$$
k=\frac{\alpha^{2}+1}{2 \alpha^{2}} k_{g}-\left(\gamma q_{1} q_{2}-\frac{\tau_{o}^{2}}{2 \alpha^{2}}\right) k_{e}-\frac{\tau_{o}}{2 \alpha^{2}} k_{s},
$$

consequently

$$
e^{2 k}=e^{\left(\frac{\alpha^{2}+1}{2 \alpha^{2}}\right) 2 k_{g}} e^{-\left(\gamma q_{1} q_{2}-\frac{\tau_{o}^{2}}{2 \alpha^{2}}\right) 2 k_{e}} e^{-\left(\frac{\tau_{o}}{2 \alpha^{2}}\right) 2 k_{s}} \equiv e^{2 K_{2} k_{g}} e^{2 K_{1} k_{e}} e^{2 K_{3} k_{s}}
$$

where $k_{g}, k_{e}$ and $k_{s}$ are solutions of the following set of equations

$$
\begin{aligned}
k_{g, z} & =\rho \lambda_{, \rho} \lambda_{, z}, \\
k_{g, \rho} & =\frac{\rho}{2}\left(\lambda_{, \rho}^{2}+\lambda_{, z}^{2}\right),
\end{aligned}
$$




$$
\begin{gathered}
k_{e, z}=\rho \tau_{, \rho} \tau_{, z}, \\
k_{e, \rho}=\frac{\rho}{2}\left(\tau_{, \rho}^{2}+\tau_{, z}^{2}\right), \\
k_{s, z}=\rho\left(\tau_{, z} \lambda_{, \rho}+\tau_{, \rho} \lambda_{, z}\right), \\
k_{s, \rho}=\rho\left(\tau_{, z} \lambda_{, z}+\tau_{, \rho} \lambda_{, \rho}\right),
\end{gathered}
$$

Integrating Eqs. (39) with $\lambda$ and $\tau$ given by Eqs. (34), it turns out that the factor $e^{2 K_{3} k_{s}}$ does not diverge neither on $u=0$ or $v=0$. Furthermore, performing an analogous analysis as in [10], it can be shown that $\tau$ contributes to the function $k_{e}$, via Eqs. (38), with the following term on $v=0$,

$$
-\frac{1}{2} d_{1}^{2} \ln \left[(z+1)^{2}-\rho^{2}\right]=-\frac{1}{2} d_{1}^{2} \ln \left(v^{m}\right)+\text { bounded terms, }
$$

which gives the desired behaviour if $K_{1} d_{1}^{2}=2-\frac{2}{m}$. Analogously, $\lambda$ contributes to the function $k_{g}$, via Eqs. (37), with the term on $u=0$ of the form

$$
-\frac{1}{2} d_{2}^{2} \ln \left[(1-z)^{2}-\rho^{2}\right]=-\frac{1}{2} d_{2}^{2} \ln \left(u^{n}\right)+\text { bounded } \quad \text { terms }
$$

which behaves properly if $K_{2} d_{2}^{2}=2-\frac{2}{n}$.

We can use solutions for $\lambda$ and $\tau$ as those given by Eq. (29) involving more terms; however, all other contributions of $\lambda$ and $\tau$ to the function $e^{2 k}$ are found to be bounded on $u=0, v=0$. Therefore, provided there exist at least two terms of the form given by Eqs. (34), in the case (i) the verification of the boundary conditions relevant to the colliding wave problem is ensured if the constants fulfill the conditions

$$
K_{1} d_{1}^{2}=2-\frac{2}{m}, \quad K_{2} d_{2}^{2}=2-\frac{2}{n} .
$$

For the case $(i i)$ the previous analysis apply when one chooses

$$
\lambda=c_{1} \operatorname{arccosh}\left[\frac{z+1}{\rho}\right]+c_{2} \operatorname{arccosh}\left[\frac{1-z}{\rho}\right]
$$

and $\tau$, for instance, can be chosen as in (34). Again, it can be shown that the term (32) does not diverge neither on $u=0$ or $v=0$. In relation to the term (33), the constants $c_{1}$ and 
$c_{2}$ can be adjusted conveniently in order to achieve an smooth matching of the solution on $u=0$ and $v=0$. The previous analysis showed that the solutions given by Eqs. (25)-(26) subjected to (40) and (27)-(28) can be interpreted as colliding wave fields.

\section{Behaviour of the Fields on the Null Boundaries}

From Eq. (22) the nonvanishing components of the electromagnetic field turn out to be

$$
\begin{aligned}
& F_{y \rho}=\frac{a_{1} a_{4}-a_{3} a_{2}}{\left(a_{1} \Sigma_{1}+a_{2} \Sigma_{2}\right)^{2}}\left\{\Sigma_{2} \Sigma_{1, \rho}-\Sigma_{1} \Sigma_{2, \rho}\right\}, \\
& F_{y z}=\frac{a_{1} a_{4}-a_{3} a_{2}}{\left(a_{1} \Sigma_{1}+a_{2} \Sigma_{2}\right)^{2}}\left\{\Sigma_{2} \Sigma_{1, z}-\Sigma_{1} \Sigma_{2, z}\right\},
\end{aligned}
$$

if we choose, for example, $\tau$ as in Eq.(34) it is straightforward to show, from Eq. (25) for case (i) and from Eq.(27) for case (ii), that $F_{\mu \nu}$ does not diverge on $u=0$ neither on $v=0$.

For the dilaton field $\Phi$, in the case (i), substituting the Eqs.(25) and (34) in Eq. (21) and taking separately the limits $u \rightarrow 0(\rho \rightarrow 1-z)$ and $v \rightarrow 0(\rho \rightarrow 1+z)$ it can be shown that $\kappa^{2}=e^{-2 \alpha \Phi}$ does not diverge on $u=0$ neither on $v=0$ and this behaviour is independent

of the constants $\tau_{o}, d_{1}, d_{2}, q_{1}, q_{2}, \alpha$. The analogous occurs for case (ii) subtituting in (21) the Eqs. (27) and (34). For the precolliding region IV $(u \leq 0, \quad v \leq 0)$, for the case (i), the dilaton field becomes a constant, $\kappa^{2}=\kappa_{o}^{2}$, while for the case (ii) the value of $\kappa$ vanishes.

\section{Singularities and Discontinuities of the Curvature along the Null Boundaries}

We now discuss briefly the behaviour of the fields on the null boundaries $u=0$ and $v=0$ in the context of the field equations. In order to do this we pass from region I to region II and III using the Penrose's procedure [11] : the continuations of the fields from region I to the remaining regions II and III, and further to IV can be achieved by replacing the coordinates $u$ and $v$ in accordance with

$$
u \rightarrow u H(u), \quad v \rightarrow v H(v)
$$

As a consequence of this procedure, singularities or discontinuities (or both) of the Riemann tensor can arise on the null hypersurfaces. To determine their behaviour we follow the 
analysis accomplished by Chandrasekhar and Xanthopoulos [12]. In their paper they showed that the quantities involving first derivatives of the metric functions can at most suffer an $\Theta$ function discontinuity, while those quantities with second derivatives in the coordinates $u$ or $v$, can involve $\delta$-function singularities. With this criteria, we can characterize the behavior of the fields, Einstein tensor components and curvature on the null boundaries.

From the field Eqs. (7)-(13) we can see that they involve first derivatives and terms of the form of mixed derivatives $\partial^{2} / \partial_{u} \partial_{v}$, but mixed derivatives do not lead to $\delta$-function distributions, then the fields are consistent on the null boundaries, provided we select $U_{\text {,uu }}$ and $U_{, v v}$ such that this second derivative do not lead to a $\delta$-function behavior. The curvature components (15)-(17) behave as it should be on the null boundaries; for a detailed general analysis in this respect see [13]

\section{Singularities on the Focussing Hypersurface}

Colliding plane wave solutions exhiibit singularities at the so called foccusing surface. The origin of this singularity has been discussed in [14]. From the metric (18), we realize that singularities can arise when $f=0$ or if $e^{2 k}$ diverges. Both behaviours can occur when $\rho=1-v^{m}-u^{n}=0$. For the case (i), we balance separately each term by arranging the constants properly. For $f$ we have ( writting only the terms which depend on $\rho$ ):

$$
\begin{aligned}
& e^{-d_{2} \ln \{\}}\left(a_{1} e^{q_{1} d_{1} \ln \{\}}+a_{2} e^{q_{2} d_{1} \ln \{\}}\right)^{\gamma} \\
& \simeq\left[\frac{1-z \pm \sqrt{(1-z)^{2}-\rho^{2}}}{\rho}\right]^{-d_{2}}\left\{a_{1}\left[\frac{z+1 \pm \sqrt{(z+1)^{2}-\rho^{2}}}{\rho}\right]^{q_{1} d_{1}}+\right. \\
& \left.a_{2}\left[\frac{1+z \pm \sqrt{(1+z)^{2}-\rho^{2}}}{\rho}\right]^{q_{2} d_{1}}\right\} \\
& \simeq \rho^{d_{2}-q_{1} d_{1} \gamma}\left[1+() \rho^{-q_{2} d_{1}+q_{1} d_{1}}\right]^{\gamma}
\end{aligned}
$$

expanding the term in brackets, we take the highest power in $\rho, \rho^{\left(-q_{2} d_{1}+q_{1} d_{1}\right) \gamma}$. This term must balance the term outside the bracket. If the constants can be adjusted in such a manner that $d_{2}-q_{1} d_{1} \gamma+\gamma\left(-q_{2} d_{1}+q_{1} d_{1}\right)>0$, or $d_{2}>q_{2} d_{1} \gamma$, then this term does not diverge at $\rho=0$. Examining now the factor $e^{2 k}$, from the integration of Eqs. (37)-(39) the terms which 
diverge at $\rho=0$ are

$$
e^{2 k} \simeq \rho^{K_{2} d_{2}^{2}+K_{1} d_{1}^{2}+2 K_{3} d_{1} d_{2}} \text { (bounded terms) }
$$

Therefore, the singularity will be avoided if we impose one more condition :

$$
K_{2} d_{2}^{2}+K_{1} d_{1}^{2}+2 K_{3} d_{1} d_{2} \geq 0
$$

Consequently, imposing on the constants the conditions determined above, the singularity can be avoided for the case (i).

For the case (ii) the term corresponding to $e^{2 k}$ can always be arranged to be not divergent, however, for $f$ we have

$$
e^{-\lambda}\left(a_{1} \tau+a_{2}\right)^{\gamma} \simeq \text { bounded terms }+() \rho^{c_{1}+c_{2}}(\ln \rho)^{a_{1} d_{1} \gamma}
$$

the last term can not be balanced with the another term, thus in this case the singularity can not be avoided.

\section{Final Remarks}

In this paper it is considered the problem of the field arising as a result of collision of plane gravitational waves in the Einstein-Maxwell-Dilaton fields. Two solutions of the Einstein-Maxwell-Dilaton equations interpretable as colliding gravitational plane waves are explicitly given. The metric is diagonal, this means that the two commuting Killing vectors are orthogonal. The verification of the boundary conditions relevant to the colliding wave problem are determined essentially by the physical structure of the incoming plane waves, whose "amplitude" must be adjusted (Eqs. (40)) depending on the values of the coupling constant $\alpha$, the constant of the dilaton field $\tau_{o}$, and the constants $q_{1}, q_{2}$ of the metric functions. For the boundary conditions the waves act separately on each boundary $u=0$ and $v=0$. It is discussed briefly the behavior of the fields on the null boundaries. In relation to the singularity developed after the waves collide, it occurs that to avoid the singularity, for our first case, it must be imposed conditions which involve both amplitudes and also the coupling constant $\alpha$. In contrast, for the case (ii), the singularity cannot be avoided by 
tuning properly the free parameters. Remains as an open question if the solutions presented here can be extended to all orders in the string tension parameter.

\section{Acknowledgements}

This work was partially supported by CONACyT (México). 


\section{References}

1. D. Garfinkle, G. Horowitz and A. Strominger, Phys. Rev. D 43, 3140 (1991); G. Gibbons and K. Maeda, Nucl. Phys. B298, 741 (1988).

2. R. Güven, Phys. Lett. B191, 275 (1987); G. Horowitz and A. Steif, Phys. Rev. Lett. 64, $260(1990)$.

3. M. Gürses and E. Sermutlu, Phys. Rev. D 52, 809 (1995)

4. J. B. Griffiths, Colliding Plane Waves in General Relativity, (Oxford: Oxford Univ. Press), 1991, Ch. 20.

5. J. H. Horne and G. T. Horowitz, Phys. Rev. D46, 1340 (1992).

6. J. H. Horne and G. T. Horowitz, Phys. Rev. D48, R5457, (1993).

7. N. Bretón and T. Matos "Colliding waves as harmonic maps" in Gravitation: The Spacetime Structure, Proceedings of the 8th Latin American Symposium on Relativity and Gravitation, Aguas de Lindoia, Brazil, 1993. ed. by P. Letelier and W. Rodriguez (World Scientificc, Singapore, 1994)

8. S. Mizoguchi. "Colliding Wave Solutions, Duality and Diagonal Embedding of General Relativity in two-dimensional Heterotic String Theory", PREPRINT DESY95-126.

9. P. Szekeres, J. Math. Phys. 13, 286, (1972); S. O'Brien and J. L. Synge, Proc. Dublin Inst. Adv. Stud. A9, 1 (1952).

10. A. Feinstein and J. Ibáñez, Phys. Rev. D39, 470, (1989).

11. K. Khan and R. Penrose, Nature (London), 229, 185 (1971).

12. S. Chandrasekhar and B. C. Xanthopoulos, Proc. R. Soc. Lond. A398, 223 (1985).

13. A. García, Theor. and Math. Phys. 83, 434 (1990).

14. R. A. Matzner and F. J. Tipler, Phys. Rev. D29, 1575, (1984). 